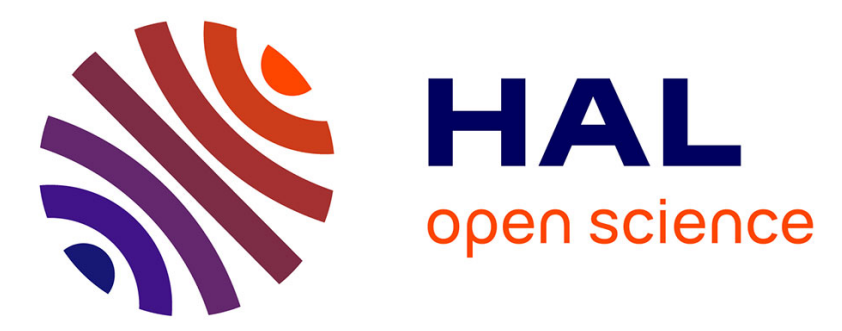

\title{
Uniqueness of Conservative Solutions to the two-component Camassa-Holm System via Characteristics
}

Li Mingjie, Zhang Qingtian

\section{- To cite this version:}

Li Mingjie, Zhang Qingtian. Uniqueness of Conservative Solutions to the two-component CamassaHolm System via Characteristics. 2015. hal-01183748

\section{HAL Id: hal-01183748 \\ https://hal.science/hal-01183748}

Preprint submitted on 11 Aug 2015

HAL is a multi-disciplinary open access archive for the deposit and dissemination of scientific research documents, whether they are published or not. The documents may come from teaching and research institutions in France or abroad, or from public or private research centers.
L'archive ouverte pluridisciplinaire HAL, est destinée au dépôt et à la diffusion de documents scientifiques de niveau recherche, publiés ou non, émanant des établissements d'enseignement et de recherche français ou étrangers, des laboratoires publics ou privés.

\section{(c) (1) $\$$}

Distributed under a Creative Commons Attribution - NonCommercial| 4.0 International 


\title{
Uniqueness of Conservative Solutions to the two-component Camassa-Holm System via Characteristics
}

\author{
Mingjie $\mathrm{Li}^{(*)}$ and Qingtian $\operatorname{Zhang}^{(* *)}$ \\ (*)College of Science, Minzu University of China, Beijing 100081, P. R. China \\ (**) Department of Mathematics, Penn State University, University Park, Pa. 16802, U.S.A. \\ e-mails: lmjmath@gmail.com, zhang_q@math.psu.edu
}

July 27, 2015

\begin{abstract}
The paper is concerned with a direct proof the uniqueness of global conservative solutions to the two-component Camassa-Holm system, based on characteristics. Given a conservative solution $u=u(t, x)$ and $\rho=\rho(t, x)$, an equation is introduced to single out a unique characteristic curve through each initial point. It is proved that the Cauchy problem with general initial data $u_{0} \in H^{1}(R), \rho_{0} \in L^{2}(R)$ has a unique global conservative solution.
\end{abstract}

\section{Introduction}

The Cauchy problem for the two-component Camassa-Holm system $(2 \mathrm{CH})$ can be written in the form

$$
\left\{\begin{array}{l}
u_{t}+\left(u^{2} / 2\right)_{x}+P_{x}=0, \\
\rho_{t}+(u \rho)_{x}=0,
\end{array}\right.
$$

where the nonlocal source term $P$ is defined as a convolution:

$$
P \doteq \frac{1}{2} e^{-|x|} *\left(u^{2}+\frac{\left(u_{x}^{2}+\rho^{2}\right)}{2}\right) .
$$

The initial data is specified as

$$
u(0, x)=u_{0}(x), \rho(0, x)=\rho_{0}(x) .
$$

The equations have been derived as a model for shallow water by Constantin and Ivanov [8]. The variable $u(t, x)$ describes the horizontal velocity of the fluid and $\rho(t, x)$ is related to the horizontal deviation of the free surface from equilibrium. Mathematical properties of this 
system and its generalized model have been studied in many works. In [19], a local solution of $2 \mathrm{CH}$ system is established in a range of the Besov spaces by Gui and Liu through LittlewoodPaley analysis. A global solution of the $2 \mathrm{CH}$ system is established by Gui and Liu [18] in the Sobolev space $H^{s}$ by using the localization analysis in the transport equation theory under the condition

$$
\inf _{x \in \mathbb{R}} \rho_{0}(x)>0 .
$$

They proved the slope of $u$ can be controlled by $\rho$ provided the sign of $\rho$ does not change.

The $2 \mathrm{CH}$ system possesses not only global-in-time solutions but also possesses wave-breaking solutions. There is a huge amount of literature showing that this system has the wave-breaking phenomenon, see $[7,8,10,11,13,16,18]$. The velocity $u(x, t)$ to the $2 \mathrm{CH}$ system remains bounded but its slope becomes unbounded in finite time $T$. This raises the natural question of the behaviour of the solutions after wave breaking. Two scenarios can be put forward. The first suggests energy loss at blow up time $T$ in the sense that

$$
E(t)<E(T)
$$

for all $t>T$ with

$$
E(t)=\int_{\mathbb{R}}\left(u^{2}(t, x)+u_{x}^{2}(t, x)+\rho^{2}(t, x)\right) d x .
$$

Whereas the second scenario proposes a switching phenomenon: the waves pass through each other, each continuing unscathed as a solitary wave (see [3] for the Camassa-Holm equation). The second scenario would correspond to energy preserving. Recently, Grunert, Holden and Raynaud [14] prove existence of a global conservative solution of the Cauchy problem for the $2 \mathrm{CH}$ system on the line, allowing for nonvanishing and distinct asymptotics at plus and minus infinity. In [15] Grunert, Holden and Raynaud introduce $\alpha$-dissipative solutions, that provides a continuous interpolation between conservative and dissipative solutions of the Cauchy problem for the $2 \mathrm{CH}$ system on the line with vanishing asymptotics.

However, the uniqueness of global weak solutions to Eq. (1.1) have not been discussed yet. The purpose of this paper is to show the uniqueness of the global conservative weak solution (see Definition 2) of the $2 \mathrm{CH}$ system by suitable modifying recent results [1] for the (scalar) Camassa-Holm $(\mathrm{CH})$ equation $[3,4,5,6,20,21,22]$

$$
u_{t}-u_{t x x}+3 u u_{x}=2 u_{x} u_{x x}+u u_{x x x} .
$$

In [1], the authors develop a direct approach to the uniqueness of conservative solutions for scalar Camassa-Holm equation based on characteristics. They extended the idea of generalized characteristics[9], or so-called energy variable to Camassa-Holm equation to separate different characteristics after singularity occurs. Then by writing a set of ODEs satisfied by $u$ and $u_{x}$ along the characteristic starting at a given point $\bar{y}$, they prove the uniqueness of conservative solutions.

For two-component Camassa-Holm equation, some new difficulties appear. Compared with $\mathrm{CH}$ (1.7) in [1], $2 \mathrm{CH}$ coupled with $\rho$ which is possibly tending to 0 . And wave breaking may happen at this stuation. This force us to add the energy of density, $\rho^{2}$ into coordinate transform, which give a resulting effect that the old technique of introducing $\arctan u_{x}$ for semilinear system does not work any more. To this end, we introduce a set of new quantities to describe the dynamics of $u_{x}$ and $\rho$ inspired by the work [2] dealing with the variational wave equation. Indeed if we introduce other variable to transform this blow up point to a regular 
one which is similar to $v:=\arctan u_{x}$, then the calculation will be tediously long. Instead, we choose the ratio of distance in space variable and energy variable, see (4.11). Then we use an algebra equation to recover $\rho$ and gradient $u_{x}$.

The remainder of the paper is organized as follows. In Section 2, we review basic notation and state our main theorem, Theorem 2. Section 3 establishes the uniqueness of characteristics. In Section 4 we study the dynamics of $x_{\beta}, u, u_{\beta}$ of a conservative solution along a characteristic, and present the proof of the main theorem.

\section{Basic definitions and results}

To make sense of the source term $P$, at each time $t$ we require that the function $u(t, \cdot)$ lies in the space $H^{1}(\mathbb{R})$ of absolutely continuous functions $u \in \mathbf{L}^{2}(\mathbb{R})$ with derivative $u_{x} \in \mathbf{L}^{2}(\mathbb{R})$, endowed with the norm

$$
\|u\|_{H^{1}} \doteq\left(\int_{\mathbb{R}}\left[u^{2}(x)+u_{x}^{2}(x)\right] d x\right)^{1 / 2} .
$$

For $u \in H^{1}(\mathbb{R})$ and $\rho \in L^{2}(\mathbb{R})$, Young's inequality ensures that

$$
P=\left(1-\partial_{x}^{2}\right)^{-1}\left(u^{2}+\frac{u_{x}^{2}+\rho^{2}}{2}\right) \in H^{1}(\mathbb{R}) .
$$

For future use we record the following inequalities, valid for any function $u \in H^{1}(\mathbb{R})$ :

$$
\begin{gathered}
\|u\|_{\mathbf{L}^{\infty}} \leq\|u\|_{H^{1}}, \\
\|P\|_{\mathbf{L}^{\infty}}, \quad\left\|P_{x}\right\|_{\mathbf{L}^{\infty}} \leq\left\|\frac{1}{2} e^{-|x|}\right\|_{\mathbf{L}^{\infty}} \cdot\left\|u^{2}+\frac{u_{x}^{2}+\rho^{2}}{2}\right\|_{\mathbf{L}^{1}} \leq \frac{1}{2}\left(\|u\|_{H^{1}}^{2}+\|\rho\|_{L^{2}}^{2}\right), \\
\|P\|_{\mathbf{L}^{2}}, \quad\left\|P_{x}\right\|_{\mathbf{L}^{2}} \leq\left\|\frac{1}{2} e^{-|x|}\right\|_{\mathbf{L}^{2}} \cdot\left\|u^{2}+\frac{u_{x}^{2}+\rho^{2}}{2}\right\|_{\mathbf{L}^{1}} \leq \frac{1}{\sqrt{2}}\left(\|u\|_{H^{1}}^{2}+\|\rho\|_{L^{2}}^{2}\right) .
\end{gathered}
$$

Definition 1. By a solution of the Cauchy problem (1.1)-(1.3) on $[0, T]$ we mean a Hölder continuous function $u=u(t, x)$ defined on $[0, T] \times \mathbb{R}$ and $\rho \in L^{\infty}\left(0, T ; L^{2}(\mathbb{R})\right)$ with the following properties. At each fixed $t$ we have $u(t, \cdot) \in H^{1}(\mathbb{R})$. Moreover, the map $t \mapsto u(t, \cdot)$ is Lipschitz continuous from $[0, T]$ into $\mathbf{L}^{2}(\mathbb{R})$ and satisfies the initial condition (1.3) together with

$$
\frac{d}{d t} u=-u u_{x}-P_{x}
$$

for a.e. t. Here (2.4) is understood as an equality between functions in $\mathbf{L}^{2}(\mathbb{R})$. And

$$
\frac{d}{d t} \rho=-(\rho u)_{x}
$$

Here (2.5) is understood in the sense of distribution.

As shown in $[8,10,13]$, as soon as the gradient of a solution blows up, uniqueness is lost, in general. To single out a unique solution, some additional conditions are needed. 
For smooth solutions, differentiating (1.1) w.r.t. $x$ one obtains

$$
u_{x t}+\left(u u_{x}\right)_{x}=\left(u^{2}+\frac{u_{x}^{2}+\rho^{2}}{2}\right)-P .
$$

Multiplying $(1.1)_{1}$ by $u,(1.1)_{2}$ by $\rho$ and $(2.6)$ by $u_{x}$, we obtain the three conservation laws with source term

$$
\begin{gathered}
\left(\frac{u^{2}}{2}\right)_{t}+\left(\frac{u^{3}}{3}+u P\right)_{x}=u_{x} P, \\
\left(\frac{u_{x}^{2}}{2}\right)_{t}+\left(\frac{u u_{x}^{2}}{2}-\frac{u^{3}}{3}\right)_{x}=\frac{\rho^{2}}{2} u_{x}-u_{x} P, \\
\left(\frac{\rho^{2}}{2}\right)_{t}+\left(\frac{\rho^{2}}{2} u\right)_{x}=-\frac{\rho^{2}}{2} u_{x}
\end{gathered}
$$

Summing (2.7) and (2.9), and integrating w.r.t. $x$, we see that for smooth solutions the total energy

$$
E(t) \doteq \int_{\mathbb{R}}\left(u^{2}(t, x)+u_{x}^{2}(t, x)+\rho^{2}(t, x)\right) d x
$$

is constant in time.

Definition 2. A solution $u=u(t, x)$ is conservative if $w=u_{x}^{2}+\rho^{2}$ provides a distributional solution to the balance law (2.11),

$$
w_{t}+(u w)_{x}=2\left(u^{2}-P\right) u_{x}
$$

namely

$$
\int_{0}^{\infty} \int\left[w \varphi_{t}+u w \varphi_{x}+2\left(u^{2}-P\right) u_{x} \varphi\right] d x d t+\int\left(u_{0, x}^{2}(x)+\rho_{0}^{2}(x)\right) \varphi(0, x) d x=0
$$

for every test function $\varphi \in \mathcal{C}_{c}^{1}\left(\mathbb{R}^{2}\right)$.

The main result proved in $[14,17,18]$, on the global existence of conservative solutions can be stated as follows.

Theorem 1. For any initial data $u_{0} \in H^{1}(\mathbb{R}), \rho_{0} \in L^{2}(\mathbb{R})$ the 2-component Camassa-Holm equation has a global conservative solution $u=u(t, x), \rho=\rho(t, x)$. More precisely, there exists a family of Radon measures $\left\{\mu_{(t)}, t \in \mathbb{R}\right\}$, depending continuously on time w.r.t. the topology of weak convergence of measures, such that the following properties hold.

(i) The functions $u$ and $\rho$ provide a solution to the Cauchy problem (1.1)-(1.3) in the sense of Definition 1.

(ii) There exists a null set $\mathcal{N} \subset \mathbb{R}$ with meas $(\mathcal{N})=0$ such that for every $t \notin \mathcal{N}$ the measure $\mu_{(t)}$ is absolutely continuous and has density $u_{x}^{2}(t, \cdot)+\rho^{2}(t, \cdot)$ w.r.t. Lebesgue measure.

(iii) The family $\left\{\mu_{(t)} ; t \in \mathbb{R}\right\}$ provides a measure-valued solution $w$ to the linear transport equation with source

$$
w_{t}+(u w)_{x}=2\left(u^{2}-P\right) u_{x} .
$$


At a time $t \in \mathcal{N}$ the measure $\mu_{(t)}$ has a nontrivial singular part. For a conservative solution $u$ which is not smooth, in general we only know that the energy $E$ in (2.10) coincides a.e. with a constant. Namely,

$$
E(t)=E(0) \quad \text { for } t \notin \mathcal{N}, \quad E(t)<E(0) \text { for } t \in \mathcal{N} \text {. }
$$

The main purpose of this paper is to prove the uniqueness of the above solution.

Theorem 2. For any initial data $u_{0} \in H^{1}(\mathbb{R})$ and $\rho_{0} \in L^{2}(\mathbb{R})$, the Cauchy problem (1.1)(1.3) has a unique conservative solution.

Remark 1. In this Theorem, we do not need

$$
\inf _{x \in \mathbb{R}} \rho_{0}(x)>0 .
$$

That means we obtain uniqueness in the basic energy space, no more regularity required.

Remark 2. By slightly modification Theorem 2 also holds for the $2 \mathrm{CH}$ system on the line with nonvanishing and distinct spatial asymptotics.

\section{Uniqueness of characteristics}

Let $u=u(t, x)$ and $\rho=\rho(t, x)$ be a solution of the Cauchy problem (1.1)-(1.3) with the additional balance law (2.12). We introduce the coordinates $(t, \beta)$, related to the original coordinates $(t, x)$ by the integral relation $(3.1)$

$$
x(t, \beta)+\int_{-\infty}^{x(t, \beta)}\left(u_{x}^{2}+\rho^{2}\right)(t, \xi) d \xi=\beta .
$$

At times $t$ where the measure $\mu_{(t)}$ is not absolutely continuous w.r.t. Lebesgue measure, we can define $x(t, \beta)$ to be the unique point $x$ such that

$$
\left.\left.x(t, \beta)+\mu_{(t)}(]-\infty, x[) \leq \beta \leq x(t, \beta)+\mu_{(t)}(]-\infty, x\right]\right) .
$$

Notice that (3.2) and (3.1) coincide at every time where $\mu_{(t)}$ is absolutely continuous with density $u_{x}^{2}+\rho^{2}$ w.r.t. Lebesgue measure. The next lemma which is a slight modification of Lemma 2 in [1], together with Lemma 3, establishes the Lipschitz continuity of $x$ and $u$ as functions of the variables $t, \beta$.

Lemma 1. Let $u=u(t, x)$ and $\rho=\rho(t, x)$ be a conservative solution of (1.1). Then, for every $t \geq 0$, the maps $\beta \mapsto x(t, \beta)$ and $\beta \mapsto u(t, \beta) \doteq u(t, x(t, \beta))$ implicitly defined by (3.2) are Lipschitz continuous with constant 1 . The map $t \mapsto x(t, \beta)$ is also Lipschitz continuous with a constant depending only on $\left\|u_{0}\right\|_{H^{1}}$ and $\left\|\rho_{0}\right\|_{L^{2}}$.

Proof. 1. Fix any time $t \geq 0$. The the map

$$
x \mapsto \beta(t, x) \doteq x+\int_{-\infty}^{x}\left[u_{x}^{2}(t, y)+\rho^{2}(t, y)\right] d y
$$


is right continuous and strictly increasing. Hence it has a well defined, continuous, nondecreasing inverse $\beta \mapsto x(t, \beta)$. If $\beta_{1}<\beta_{2}$, then

$$
x\left(t, \beta_{2}\right)-x\left(t, \beta_{1}\right)+\mu_{(t)}(] x\left(t, \beta_{1}\right), x\left(t, \beta_{2}\right)[) \leq \beta_{2}-\beta_{1} .
$$

This implies

$$
x\left(t, \beta_{2}\right)-x\left(t, \beta_{1}\right) \leq \beta_{2}-\beta_{1},
$$

showing that the map $\beta \mapsto x(t, \beta)$ is a contraction.

2. To prove the Lipschitz continuity of the map $\beta \mapsto u(t, \beta)$, assume $\beta_{1}<\beta_{2}$. By (3.3) it follows

$$
\begin{gathered}
\left|u\left(t, x\left(t, \beta_{2}\right)\right)-u\left(t, x\left(t, \beta_{1}\right)\right)\right| \leq \int_{x\left(t, \beta_{1}\right)}^{x\left(t, \beta_{2}\right)}\left|u_{x}\right| d y \leq \int_{x\left(t, \beta_{1}\right)}^{x\left(t, \beta_{2}\right)} \frac{1}{2}\left(1+u_{x}^{2}\right) d y \\
\leq \frac{1}{2}\left[x\left(t, \beta_{2}\right)-x\left(t, \beta_{1}\right)+\mu_{(t)}(] x\left(t, \beta_{1}\right), x\left(t, \beta_{2}\right)[)\right] \leq \frac{1}{2}\left(\beta_{2}-\beta_{1}\right) .
\end{gathered}
$$

3. Next, we prove the Lipschitz continuity of the map $t \mapsto x(t, \beta)$. Assume $x(\tau, \beta)=y$. We recall that the family of measures $\mu_{(t)}$ satisfies the balance law (2.13), where for each $t$ the drift $u$ and the source term $2\left(u^{2}-P\right) u_{x}$ satisfy

$$
\begin{gathered}
\|u\|_{\mathbf{L}^{\infty}(\mathbb{R})} \leq C_{\infty} \doteq\|u\|_{H^{1}(\mathbb{R})}, \\
\left\|2\left(u^{2}-P\right) u_{x}\right\|_{\mathbf{L}^{1}(\mathbb{R})} \leq 2\left(\|u\|_{\mathbf{L}^{\infty}}\|u\|_{\mathbf{L}^{2}}+\|P\|_{\mathbf{L}^{2}}\right)\left\|u_{x}\right\|_{\mathbf{L}^{2}} \leq C_{S},
\end{gathered}
$$

for some constant $C_{S}$ depending only on the $H^{1}$ norm of $u$ and $L^{2}$ norm of $\rho$. Therefore, for $t>\tau$ we have

$$
\begin{aligned}
\mu_{(t)}(]-\infty, y-C_{\infty}(t-\tau)[) & \leq \mu_{(\tau)}(]-\infty, y[)+\int_{\tau}^{t}\left\|2\left(u^{2}-P\right) u_{x}\right\|_{\mathbf{L}^{1}(\mathbb{R})} d s \\
& \leq \mu_{(\tau)}(]-\infty, y[)+C_{S}(t-\tau) .
\end{aligned}
$$

Defining $y^{-}(t) \doteq y-\left(C_{\infty}+C_{S}\right)(t-\tau)$, we obtain

$$
\begin{aligned}
& y^{-}(t)+\mu_{(t)}(]-\infty, y^{-}(t)[) \leq y-\left(C_{\infty}+C_{S}\right)(t-\tau)+\mu_{(\tau)}(]-\infty, y[)+C_{S}(t-\tau) \\
& \leq y+\mu_{(\tau)}(]-\infty, y[) \leq \beta .
\end{aligned}
$$

This implies $x(t, \beta) \geq y^{-}(t)$ for all $t>\tau$. An entirely similar argument yields $x(t, \beta) \leq y^{+}(t) \doteq$ $y+\left(C_{\infty}+C_{S}\right)(t-\tau)$, proving the uniform Lipschitz continuity of the map $t \mapsto x(t, \beta)$. 
The next result, which provides the foundation to all our analysis, shows that characteristics can be uniquely determined by $\beta(t)$, which is the combination of (3.7) with (3.8),

$$
\begin{gathered}
\frac{d}{d t} x(t)=u(t, x(t)), \quad x(0)=\bar{y} \\
\frac{d}{d t} \int_{-\infty}^{x(t, \beta)}\left(u_{x}^{2}+\rho^{2}\right) d x=\int_{-\infty}^{x(t, \beta)}\left[2 u^{2}-2 P\right] u_{x}(t, x) d x
\end{gathered}
$$

at the time $t \notin \mathcal{N}$.

Lemma 2. Let $u=u(t, x)$ be a conservative solution of the Camassa-Holm equation. Then, for any $\bar{y} \in \mathbb{R}$ there exists a unique Lipschitz continuous map $t \mapsto x(t)$ which satisfies both (3.7) and (3.8). In addition, for any $0 \leq \tau \leq t$ one has

$$
u(t, x(t))-u(\tau, x(\tau))=-\int_{\tau}^{t} P_{x}(s, x(s)) d s .
$$

Proof. 1. Using the adapted coordinates $(t, \beta)$ as in (3.1), we write the characteristic starting at $\bar{y}$ in the form $t \mapsto x(t)=x(t, \beta(t))$, where $\beta(\cdot)$ is a map to be determined. By summing the two equations (3.7) and (3.8) and integrating w.r.t. time we obtain for $t \notin \mathcal{N}$,

$$
\begin{aligned}
x(t)+\int_{-\infty}^{x(t)}\left(u_{x}^{2}+\rho^{2}\right)(t, y) d y=\quad & +\int_{-\infty}^{\bar{y}}\left[u_{0, x}^{2}(y)+\rho_{0}^{2}(y)\right] d y \\
& +\int_{0}^{t}\left(u(s, x(s))+\int_{-\infty}^{x(s)}\left[2 u^{2} u_{x}-2 P u_{x}\right](s, y) d y\right) d s .
\end{aligned}
$$

Introducing the function

$$
G(t, \beta) \doteq \int_{-\infty}^{x(t, \beta)}\left[u_{x}+2 u^{2} u_{x}-2 u_{x} P\right] d x
$$

and the constant

$$
\bar{\beta}=\bar{y}+\int_{-\infty}^{\bar{y}}\left[u_{0, x}^{2}(y)+\rho_{0}^{2}(y)\right] d y,
$$

we can define the dynamics of $\beta(t)$ in the form

$$
\beta(t)=\bar{\beta}+\int_{0}^{t} G(s, \beta(s)) d s, \quad \text { for all } t>0 .
$$

Remark 3. We emphasis that here $\beta(t)$ is defined for every $t>0$. When $t \notin \mathcal{N}$, it is same as (3.10). However, when $\mu_{(t)}$ has nontrivial singular part, $\beta$ will play an important role. At this time, different characteristics may have same $x$ value but different $\beta$ value. In the right-hand-side of the equation (3.13), we don't need to worry about the singular measure because $\mathcal{N}$ is a measure-zero set.

2. For each fixed $t \geq 0$, since the maps $x \mapsto u(t, x)$ and $x \mapsto P(t, x)$ are both in $H^{1}(\mathbb{R})$, the function $\beta \mapsto G(t, \beta)$ defined at (3.11) is uniformly bounded and absolutely continuous. 
Moreover,

$$
G_{\beta}=\left[u_{x}+2 u^{2} u_{x}-2 u_{x} P\right] x_{\beta}=\frac{u_{x}+2 u^{2} u_{x}-2 u_{x} P}{1+u_{x}^{2}+\rho^{2}} \in[-C, C]
$$

for some constant $C$ depending only on the $H^{1}$ norm of $u$ and $L^{2}$ norm of $\rho$. Hence the function $G$ in (3.11) is uniformly Lipschitz continuous w.r.t. $\beta$.

3. Thanks to the Lipschitz continuity of the function $G$, the existence of a unique solution to the integral equation (3.13) can be proved by a standard fixed point argument. Namely, consider the Banach space of all continuous functions $\beta: \mathbb{R}_{+} \mapsto \mathbb{R}$ with weighted norm

$$
\|\beta\|_{*} \doteq \sup _{t \geq 0} e^{-2 C t}|\beta(t)| .
$$

On this space, we claim that the Picard map

$$
(\mathcal{P} \beta)(t) \doteq \bar{\beta}+\int_{0}^{t} G(\tau, \beta(\tau)) d \tau
$$

is a strict contraction. Indeed, assume $\|\beta-\tilde{\beta}\|_{*}=\delta>0$. This implies

$$
|\beta(\tau)-\tilde{\beta}(\tau)| \leq \delta e^{2 C \tau} \quad \text { for all } \tau \geq 0 .
$$

Hence

$$
\begin{aligned}
|(\mathcal{P} \beta)(t)-(\mathcal{P} \tilde{\beta})(t)| & =\left|\int_{0}^{t}(G(\tau, \beta(\tau))-G(\tau, \tilde{\beta}(\tau))) d \tau\right| \leq \int_{0}^{t} C|\beta(\tau)-\tilde{\beta}(\tau)| d \tau \\
& \leq \int_{0}^{t} C \delta e^{2 C \tau} d \tau \leq \frac{\delta}{2} e^{2 C t}
\end{aligned}
$$

We thus conclude that $\|\mathcal{P} \beta-\mathcal{P} \tilde{\beta}\|_{*} \leq \delta / 2$.

By the contraction mapping principle, the integral equation (3.13) has a unique solution.

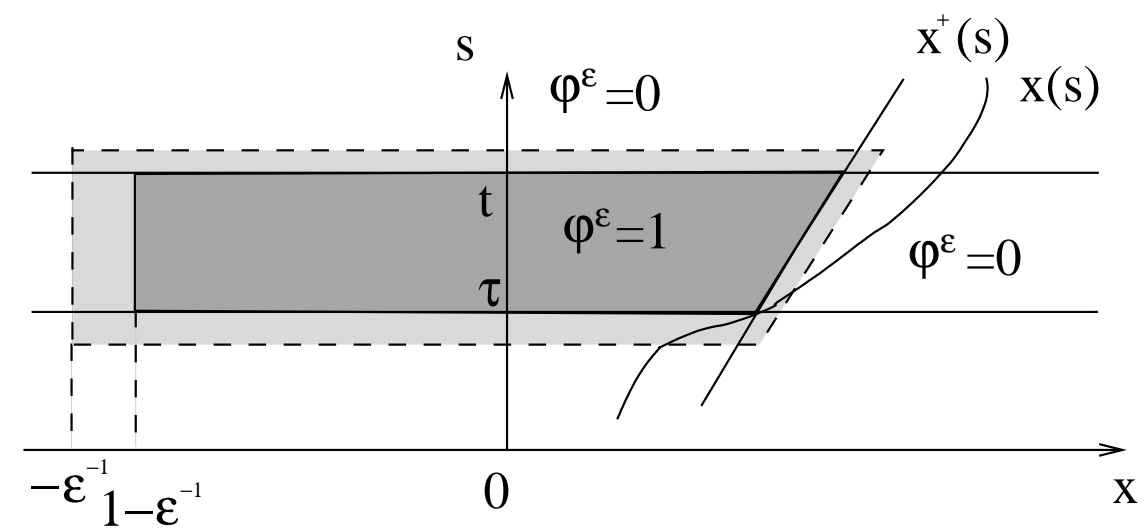

Figure 1: The Lipschitz continuous test function $\varphi^{\epsilon}$ introduced at (3.18).

4. By the previous construction, the map $t \mapsto x(t) \doteq x(t, \beta(t))$ provides the unique solution to (3.10). Being the composition of two Lipschitz functions, the map $t \mapsto x(t, \beta(t))$ is Lipschitz continuous. To prove that it satisfies the ODE for characteristics (3.7), it suffices to show that (3.7) holds at each time $\tau>0$ such that 
(i) $x(\cdot)$ is differentiable at $t=\tau$, and

(ii) the measure $\mu_{(\tau)}$ is absolutely continuous.

Assume, on the contrary, that $\dot{x}(\tau) \neq u(\tau, x(\tau))$. To fix the ideas, let

$$
\dot{x}(\tau)=u(\tau, x(\tau))+2 \epsilon_{0}
$$

for some $\epsilon_{0}>0$. The case $\epsilon_{0}<0$ is entirely similar. To derive a contradiction we observe that, for all $t \in] \tau, \tau+\delta]$, with $\delta>0$ small enough one has

$$
x^{+}(t) \doteq x(\tau)+(t-\tau)\left[u(\tau, x(\tau))+\epsilon_{0}\right]<x(t) .
$$

We also observe that, since $u, P$ are continuous while $u_{x} \in \mathbf{L}^{2}$, by an approximation argument the identity in (2.12) remains valid for any test function $\varphi \in H^{1}$ with compact support. In particular, this is true if $\varphi$ is Lipschitz continuous with compact support.

For any $\varepsilon>0$ small, we can thus consider the functions

$$
\begin{aligned}
& \rho^{\varepsilon}(s, y) \doteq\left\{\begin{array}{cl}
0 & \text { if } y \leq-\varepsilon^{-1}, \\
\left(y+\varepsilon^{-1}\right) & \text { if }-\varepsilon^{-1} \leq y \leq 1-\varepsilon^{-1}, \\
1 & \text { if } 1-\varepsilon^{-1} \leq y \leq x^{+}(s), \\
1-\varepsilon^{-1}(y-x(s)) & \text { if } x^{+}(s) \leq y \leq x(s)^{+}+\varepsilon \\
0 & \text { if } y \geq x^{+}(s)+\varepsilon,
\end{array}\right. \\
& \chi^{\varepsilon}(s) \doteq\left\{\begin{array}{cl}
0 & \text { if } s \leq \tau-\varepsilon \\
\varepsilon^{-1}(s-\tau+\varepsilon) & \text { if } \tau-\varepsilon \leq s \leq \tau, \\
1 & \text { if } \tau \leq s \leq t \\
1-\varepsilon^{-1}(s-t) & \text { if } t \leq s<t+\varepsilon \\
0 & \text { if } s \geq t+\varepsilon .
\end{array}\right.
\end{aligned}
$$

Define

$$
\varphi^{\varepsilon}(s, y) \doteq \min \left\{\varrho^{\varepsilon}(s, y), \chi^{\varepsilon}(s)\right\}
$$

Using $\varphi^{\varepsilon}$ as test function in (2.12) we obtain

$$
\iint\left[\left(u_{x}^{2}+\rho^{2}\right) \varphi_{t}^{\varepsilon}+u\left(u_{x}^{2}+\rho^{2}\right) \varphi_{x}^{\varepsilon}+2\left(u^{2}-P\right) u_{x} \varphi^{\varepsilon}\right] d x d t=0 .
$$

We now observe that, if $t$ is sufficiently close to $\tau$, then

$$
\lim _{\varepsilon \rightarrow 0} \int_{\tau}^{t} \int_{x^{+}(s)-\varepsilon}^{x^{+}(s)+\varepsilon}\left(u_{x}^{2}+\rho^{2}\right)\left(\varphi_{t}^{\varepsilon}+u \varphi_{x}^{\varepsilon}\right) d y d s \geq 0 .
$$

Indeed, for $s \in[\tau+\varepsilon, t-\varepsilon]$ one has

$$
0=\varphi_{t}^{\varepsilon}+\left[u(\tau, x(\tau))+\epsilon_{0}\right] \varphi_{x}^{\varepsilon} \leq \varphi_{t}^{\varepsilon}+u(s, x) \varphi_{x}^{\varepsilon},
$$

because $u(s, x)<u(\tau, x(\tau))+\epsilon_{0}$ and $\varphi_{x}^{\varepsilon} \leq 0$. 
Since the family of measures $\mu_{(t)}$ depends continuously on $t$ in the topology of weak convergence, taking the limit of (3.19) as $\varepsilon \rightarrow 0$, for $\tau, t \notin \mathcal{N}$ we obtain

$$
\begin{aligned}
0= & \int_{-\infty}^{x(\tau)}\left(u_{x}^{2}+\rho^{2}\right)(\tau, x) d y-\int_{-\infty}^{x^{+}(t)}\left(u_{x}^{2}+\rho^{2}\right)(t, y) d y+\int_{\tau}^{t} \int_{-\infty}^{x^{+}(s)}\left[2 u^{2} u_{x}-2 u_{x} P\right] d y d s \\
& \quad+\lim _{\varepsilon \rightarrow 0} \int_{\tau}^{t} \int_{x^{+}(s)-\varepsilon}^{x^{+}(s)+\varepsilon}\left(u_{x}^{2}+\rho^{2}\right)\left(\varphi_{t}^{\varepsilon}+u \varphi_{x}^{\varepsilon}\right) d y d s \\
\geq & \int_{-\infty}^{x(\tau)}\left(u_{x}^{2}+\rho^{2}\right)(\tau, y) d y-\int_{-\infty}^{x^{+}(t)}\left(u_{x}^{2}+\rho^{2}\right)(t, y) d y+\int_{\tau}^{t} \int_{-\infty}^{x^{+}(s)}\left[2 u^{2} u_{x}-2 u_{x} P\right] d y d s
\end{aligned}
$$

In turn, (3.21) implies

$$
\begin{aligned}
& \int_{-\infty}^{x^{+}(t)}\left(u_{x}^{2}+\rho^{2}\right)(t, x) d y \geq \int_{-\infty}^{x(\tau)}\left(u_{x}^{2}+\rho^{2}\right)(\tau, x) d y+\int_{\tau}^{t} \int_{-\infty}^{x^{+}(s)}\left[2 u^{2} u_{x}-2 u_{x} P\right] d y d s \\
& =\int_{-\infty}^{x(\tau)}\left(u_{x}^{2}+\rho^{2}\right)(t, y) d y+\int_{\tau}^{t} \int_{-\infty}^{x(s)}\left[2 u^{2} u_{x}-2 u_{x} P\right] d y d s+o_{1}(t-\tau) .
\end{aligned}
$$

Notice that the last term is a higher order infinitesimal, satisfying $\frac{o_{1}(t-\tau)}{t-\tau} \rightarrow 0$ as $t \rightarrow \tau$. Indeed

$$
\begin{aligned}
& \left|o_{1}(t-\tau)\right|=\left|\int_{\tau}^{t} \int_{x^{+}(s)}^{x(s)}\left[2 u^{2} u_{x}-2 P u_{x}\right] d x d s\right| \leq \int_{\tau}^{t} \int_{x^{+}(s)}^{x(s)}\left|2 u^{2}-2 P\right|\left|u_{x}\right| d y d s \\
& \quad \leq 2\left\|u^{2}-P\right\|_{\mathbf{L}^{\infty}} \cdot \int_{\tau}^{t} \int_{x^{+}(s)}^{x(s)}\left|u_{x}\right| d y d s \\
& \quad \leq 2\left\|u^{2}-P\right\|_{\mathbf{L}^{\infty}} \int_{\tau}^{t}\left(x(s)-x^{+}(s)\right)^{1 / 2}\left\|u_{x}(s, \cdot)\right\|_{\mathbf{L}^{2}} d s \leq C \cdot(t-\tau)^{3 / 2} .
\end{aligned}
$$

On the other hand, by (3.11) and (3.13) a linear approximation yields

$$
\beta(t)=\beta(\tau)+(t-\tau)\left[u(\tau, x(\tau))+\frac{2}{3} u^{3}(\tau, x(\tau))-\int_{-\infty}^{x(\tau)} 2 u_{x} P d y\right]+o_{2}(t-\tau),
$$

with $\frac{o_{2}(t-\tau)}{t-\tau} \rightarrow 0$ as $t \rightarrow \tau$. 
For every $t>\tau$ with $t \notin \mathcal{N}, t$ sufficiently close to $\tau$, we now have

$$
\begin{aligned}
\beta(t) & =x(t)+\int_{-\infty}^{x(t)}\left(u_{x}^{2}+\rho^{2}\right)(t, y) d y \\
> & x(\tau)+(t-\tau)\left[u(\tau, x(\tau))+\epsilon_{0}\right]+\int_{-\infty}^{x^{+}(t)}\left(u_{x}^{2}+\rho^{2}\right)(t, y) d y \\
\geq & x(\tau)+(t-\tau)\left[u(\tau, x(\tau))+\epsilon_{0}\right]+\int_{-\infty}^{x(\tau)}\left(u_{x}^{2}+\rho^{2}\right)(\tau, y) d y \\
& \quad+\int_{\tau}^{t} \int_{-\infty}^{x(s)}\left[2 u^{2} u_{x}-2 u_{x} P\right] d y d s+o_{1}(t-\tau) .
\end{aligned}
$$

Combining (3.22) and (3.23), we find

$$
\begin{aligned}
\beta(\tau)+( & t-\tau)\left[u(\tau, x(\tau))+\int_{-\infty}^{x(\tau)}\left[2 u^{2} u_{x}-2 u_{x} P\right] d y\right]+o_{2}(t-\tau) \\
\geq & {\left[x(\tau)+\int_{-\infty}^{x(\tau)}\left(u_{x}^{2}+\rho^{2}\right)(\tau, y) d y\right]+(t-\tau)\left[u(\tau, x(\tau))+\epsilon_{0}\right] } \\
& +\int_{\tau}^{t} \int_{-\infty}^{x(s)}\left[2 u^{2} u_{x}-2 u_{x} P\right] d y d s+o_{1}(t-\tau) .
\end{aligned}
$$

Subtracting common terms, dividing both sides by $t-\tau$ and letting $t \rightarrow \tau$, we achieve a contradiction. Therefore, (3.7) must hold.

5. We now prove (3.9). By (2.4), for every test function $\phi \in \mathcal{C}_{c}^{\infty}\left(\mathbb{R}^{2}\right)$ one has

$$
\int_{0}^{\infty} \int\left[u \phi_{t}+\frac{u^{2}}{2} \phi_{x}+P_{x} \phi\right] d x d t+\int u_{0}(x) \phi(0, x) d x=0 .
$$

Given any $\varphi \in \mathcal{C}_{c}^{\infty}$, let $\phi=\varphi_{x}$. Since the map $x \mapsto u(t, x)$ is absolutely continuous, we can integrate by parts w.r.t. $x$ and obtain

$$
\int_{0}^{\infty} \int\left[u_{x} \varphi_{t}+u u_{x} \varphi_{x}+P_{x} \varphi_{x}\right] d x d t+\int u_{0, x}(x) \varphi(0, x) d x=0 .
$$

By an approximation argument, the identity (3.26) remains valid for any test function $\varphi$ which is Lipschitz continuous with compact support. For any $\varepsilon>0$ sufficiently small, we thus consider the functions

$$
\varrho^{\varepsilon}(s, y) \doteq\left\{\begin{array}{cl}
0 & \text { if } y \leq-\varepsilon^{-1} \\
y+\varepsilon^{-1} & \text { if }-\varepsilon^{-1} \leq y \leq 1-\varepsilon^{-1} \\
1 & \text { if } 1-\varepsilon^{-1} \leq y \leq x(s) \\
1-\varepsilon^{-1}(y-x(s)) & \text { if } x(s) \leq y \leq x(s)+\varepsilon \\
0 & \text { if } y \geq x(s)+\varepsilon
\end{array}\right.
$$




$$
\psi^{\varepsilon}(s, y) \doteq \min \left\{\varrho^{\varepsilon}(s, y), \chi^{\varepsilon}(s)\right\},
$$

where $\chi^{\varepsilon}(s)$ as in (3.17). We now use the test function $\varphi=\psi^{\varepsilon}$ in (3.26) and let $\varepsilon \rightarrow 0$. Observing that the function $P_{x}$ is continuous, we obtain

$$
\begin{aligned}
\int_{-\infty}^{x(t)} u_{x}(t, y) d y & =\int_{-\infty}^{x(\tau)} u_{x}(\tau, y) d y-\int_{\tau}^{t} P_{x}(s, x(s)) d s \\
& +\lim _{\varepsilon \rightarrow 0} \int_{\tau-\varepsilon}^{t+\varepsilon} \int_{x(s)}^{x(s)+\varepsilon} u_{x}\left(\psi_{t}^{\varepsilon}+u \psi_{x}^{\varepsilon}\right) d y d s .
\end{aligned}
$$

To complete the proof it suffices to show that the last term on the right hand side of (3.28) vanishes. Since $u_{x} \in \mathbf{L}^{2}$, Cauchy's inequality yields

$$
\left|\int_{\tau}^{t} \int_{x(s)}^{x(s)+\varepsilon} u_{x}\left(\psi_{t}^{\varepsilon}+u \psi_{x}^{\varepsilon}\right) d y d s\right| \leq \int_{\tau}^{t}\left(\int_{x(s)}^{x(s)+\varepsilon}\left|u_{x}\right|^{2} d y\right)^{1 / 2}\left(\int_{x(s)}^{x(s)+\varepsilon}\left(\psi_{t}^{\varepsilon}+u \psi_{x}^{\varepsilon}\right)^{2} d y\right)^{1 / 2} d s .
$$

For each $\varepsilon>0$ consider the function

$$
\eta_{\varepsilon}(s) \doteq\left(\sup _{x \in \mathbb{R}} \int_{x}^{x+\varepsilon} u_{x}^{2}(s, y) d y\right)^{1 / 2}
$$

Observe that all functions $\eta_{\varepsilon}$ are uniformly bounded. Moreover, as $\varepsilon \rightarrow 0$ we have $\eta_{\varepsilon}(t) \downarrow 0$ pointwise at a.e. time $t$. Therefore, by the dominated convergence theorem,

$$
\lim _{\varepsilon \rightarrow 0} \int_{\tau}^{t}\left(\int_{x(s)}^{x(s)+\varepsilon} u_{x}^{2}(s, y) d y\right)^{1 / 2} d s \leq \lim _{\varepsilon \rightarrow 0} \int_{\tau}^{t} \eta_{\varepsilon}(s) d s=0 .
$$

On the other hand, for every time $s \in[\tau, t]$ by construction we have

$$
\psi_{x}^{\varepsilon}(s, y)=\varepsilon^{-1}, \quad \psi_{t}^{\varepsilon}(s, y)+u(s, x(s)) \psi_{x}^{\varepsilon}(s, y)=0 \quad \text { for } \quad x(s)<y<x(s)+\varepsilon .
$$

This implies

$$
\begin{aligned}
& \int_{x(s)}^{x(s)+\varepsilon}\left|\psi_{t}^{\varepsilon}(s, y)+u(s, y) \psi_{x}^{\varepsilon}(s, y)\right|^{2} d y=\varepsilon^{-2} \int_{x(s)}^{x(s)+\varepsilon}|u(s, y)-u(s, x(s))|^{2} d y \\
& \leq \varepsilon^{-1} \cdot\left(\max _{x(s) \leq y \leq x(s)+\varepsilon}|u(s, y)-u(s, x(s))|\right)^{2} \leq \varepsilon^{-1} \cdot\left(\int_{x(s)}^{x(s)+\varepsilon}\left|u_{x}(s, y)\right| d y\right)^{2} \\
& \leq \varepsilon^{-1} \cdot\left(\varepsilon^{1 / 2} \cdot\left\|u_{x}(s)\right\|_{\mathbf{L}^{2}}\right)^{2} \leq\|u(s)\|_{H^{1}}^{2} .
\end{aligned}
$$

Together, (3.31) and (3.32) prove that the integral in (3.29) approaches zero as $\varepsilon \rightarrow 0$. We 
now estimate the integral near the corners of the domain:

$$
\begin{aligned}
& \left|\left(\int_{\tau-\varepsilon}^{\tau}+\int_{t}^{t+\varepsilon}\right) \int_{x(s)}^{x(s)+\kappa \varepsilon} u_{x}\left(\psi_{t}^{\varepsilon}+u \psi_{x}^{\varepsilon}\right) d y d s\right| \\
& \quad \leq\left(\int_{\tau-\varepsilon}^{\tau}+\int_{t}^{t+\varepsilon}\right)\left(\int_{x(s)}^{x(s)+\varepsilon}\left|u_{x}\right|^{2} d y\right)^{1 / 2}\left(\int_{x(s)}^{x(s)+\varepsilon}\left(\psi_{t}^{\varepsilon}+u \psi_{x}^{\varepsilon}\right)^{2} d y\right)^{1 / 2} d s \\
& \quad \leq 2 \varepsilon \cdot\|u(s)\|_{H^{1}} \cdot\left(\int_{x(s)}^{x(s)+\varepsilon} 4 \varepsilon^{-2}\left(1+\|u\|_{\mathbf{L}^{\infty}}\right)^{2} d y\right)^{1 / 2} \leq C \varepsilon^{1 / 2} \rightarrow 0
\end{aligned}
$$

as $\varepsilon \rightarrow 0$. The above analysis has shown that

$$
\lim _{\varepsilon \rightarrow 0} \int_{\tau-\varepsilon}^{t+\varepsilon} \int_{x(s)}^{x(s)+\varepsilon} u_{x}\left(\psi_{t}^{\varepsilon}+u \psi_{x}^{\varepsilon}\right) d y d s=0 .
$$

Therefore from (3.28) we recover (3.9).

6. Finally, we prove uniqueness of $x(t)$. Assume for there are different $x_{1}(t)$ and $x_{2}(t)$, both satisfying (3.8) together with the characteristic equation (3.7). Choose Lipschitz continuous functions $\beta_{1}(t)$ and $\beta_{2}(t)$ so that $x_{1}(t)=x\left(t, \beta_{1}(t)\right)$ and $x_{2}(t)=x\left(t, \beta_{2}(t)\right)$. Then $\beta_{1}(\cdot)$ and $\beta_{2}(\cdot)$ satisfy (3.13) with the same initial condition. This contradicts with the uniqueness of $\beta$ proved in step 3 .

Relying on (3.9) we can now show the Lipschitz continuity of $u$ w.r.t. $t$, in the auxiliary coordinate system.

Lemma 3. Let $u=u(t, x)$ be a conservative solution of (1.1). Then the map $(t, \beta) \mapsto$ $u(t, \beta) \doteq u(t, x(t, \beta))$ is Lipschitz continuous, with a constant depending only on the norm $\left\|u_{0}\right\|_{H^{1}}$ and $\left\|\rho_{0}\right\|_{L^{2}}$.

Proof. Using (3.4), (3.13), and (3.9), and we obtain

$$
\begin{aligned}
|u(t, x(t, \bar{\beta}))-u(\tau, \bar{\beta})| & \leq|u(t, x(t, \bar{\beta}))-u(t, x(t, \beta(t)))|+|u(t, x(t, \beta(t)))-u(\tau, x(\tau, \beta(\tau)))| \\
& \leq \frac{1}{2}|\beta(t)-\bar{\beta}|+(t-\tau)\left\|P_{x}\right\|_{\mathbf{L}^{\infty}} \leq(t-\tau) \cdot\left(\frac{1}{2}\|G\|_{\mathbf{L}^{\infty}}+\left\|P_{x}\right\|_{\mathbf{L}^{\infty}}\right) .
\end{aligned}
$$

The next result shows that the solutions $\beta(\cdot)$ of $(3.13)$ depend Lipschitz continuously on the initial data.

Lemma 4. Let $u$ be a conservative solution to (1.1). Call $t \mapsto \beta(t ; \tau, \bar{\beta})$ the solution to the integral equation

$$
\beta(t)=\bar{\beta}+\int_{\tau}^{t} G(\tau, \beta(\tau)) d \tau
$$


with $G$ as in (3.11). Then there exists a constant $C$ such that, for any two initial data $\bar{\beta}_{1}, \bar{\beta}_{2}$ and any $t, \tau \geq 0$ the corresponding solutions satisfy

$$
\left|\beta\left(t ; \tau, \bar{\beta}_{1}\right)-\beta\left(t ; \tau, \bar{\beta}_{2}\right)\right| \leq e^{C|t-\tau|}\left|\bar{\beta}_{1}-\bar{\beta}_{2}\right| .
$$

Proof. Assume $\tau<t$. By (3.34) it follows

$$
\begin{aligned}
\left|\beta\left(t ; \tau, \bar{\beta}_{1}\right)-\beta\left(t ; \tau, \bar{\beta}_{2}\right)\right| & =\left|\bar{\beta}_{1}-\bar{\beta}_{2}+\int_{\tau}^{t} G\left(s, \beta\left(s ; \tau, \bar{\beta}_{1}\right)\right)-G\left(s, \beta\left(s ; \tau, \bar{\beta}_{2}\right)\right) d s\right| \\
& \leq\left|\bar{\beta}_{1}-\bar{\beta}_{2}\right|+\int_{\tau}^{t}\left|G\left(s, \beta\left(s ; \tau, \bar{\beta}_{1}\right)\right)-G\left(s, \beta\left(s ; \tau, \bar{\beta}_{2}\right)\right)\right| d s \\
& \leq\left|\bar{\beta}_{1}-\bar{\beta}_{2}\right|+C \int_{\tau}^{t}\left|\beta\left(s ; \tau, \bar{\beta}_{1}\right)-\beta\left(s ; \tau, \bar{\beta}_{2}\right)\right| d s \\
& \leq\left|\bar{\beta}_{1}-\bar{\beta}_{2}\right| e^{C(t-\tau)},
\end{aligned}
$$

where the last inequality is obtained using Gronwall's lemma. The case $t<\tau$ is entirely similar.

Lemma 5. Assume $u \in H^{1}(\mathbb{R}), \rho \in L^{2}(\mathbb{R})$ and define the convolution $P$ as in (1.2). Then $P_{x}$ is absolutely continuous and satisfies

$$
P_{x x}=P-\left(u^{2}+\frac{1}{2}\left(u_{x}^{2}+\rho^{2}\right)\right) .
$$

Proof. The function $\phi(x)=e^{-|x|} / 2$ satisfies the distributional identity

$$
D_{x}^{2} \phi=\phi-\delta_{0}
$$

where $\delta_{0}$ denotes a unit Dirac mass at the origin. Therefore, for every function $f \in \mathbf{L}^{1}(\mathbb{R})$, the convolution satisfies

$$
D_{x}^{2}(\phi * f)=\phi * f-f .
$$

Choosing $f=u^{2}+\left(u_{x}^{2}+\rho^{2}\right) / 2$ we obtain the result.

\section{Proof of Theorem 2}

The proof will be worked out in several steps.

1. By Lemmas 1 and 3 , the map $(t, \beta) \mapsto(x, u)(t, \beta)$ is Lipschitz continuous. An entirely similar argument shows that the maps $\beta \mapsto G(t, \beta) \doteq G(t, x(t, \beta))$ and $\beta \mapsto P_{x}(t, \beta) \doteq$ $P_{x}(t, x(t, \beta))$ are also Lipschitz continuous. By Rademacher's theorem [12], the partial derivatives $x_{t}, x_{\beta}, u_{t}, u_{\beta}, G_{\beta}$, and $P_{x, \beta}$ exist almost everywhere. Moreover, a.e. point $(t, \beta)$ is a Lebesgue point for these derivatives. Calling $t \mapsto \beta(t, \bar{\beta})$ the unique solution to the integral equation (3.13), by Lemma 4 for a.e. $\bar{\beta}$ the following holds. 
(GC) For a.e. $t>0$, the point $(t, \beta(t, \bar{\beta}))$ is a Lebesgue point for the partial derivatives $x_{t}, x_{\beta}, u_{t}, u_{\beta}, G_{\beta}, P_{x, \beta}$. Moreover, $x_{\beta}(t, \beta(t, \bar{\beta}))>0$ for a.e. $t>0$.

If (GC) holds, we then say that $t \mapsto \beta(t, \bar{\beta})$ is a good characteristic.

2. We seek an ODE describing how the quantities $u_{\beta}$ and $x_{\beta}$ vary along a good characteristic. As in Lemma 4 , we denote by $t \mapsto \beta(t ; \tau, \bar{\beta})$ the solution to (3.34). If $\tau, t \notin \mathcal{N}$, assuming that $\beta(\cdot ; \tau, \bar{\beta})$ is a $\operatorname{good}$ characteristic, differentiating (3.34) w.r.t. $\bar{\beta}$ we find

$$
\frac{\partial}{\partial \bar{\beta}} \beta(t ; \tau, \bar{\beta})=1+\int_{\tau}^{t} G_{\beta}(s, \beta(s ; \tau, \bar{\beta})) \cdot \frac{\partial}{\partial \bar{\beta}} \beta(s ; \tau, \bar{\beta}) d s
$$

Next, differentiating w.r.t. $\bar{\beta}$ the identity

$$
x(t, \beta(t ; \tau, \bar{\beta}))=x(\tau, \bar{\beta})+\int_{\tau}^{t} u(s, x(s, \beta(t ; \tau, \bar{\beta}))) d s
$$

we obtain

$$
x_{\beta}(t, \beta(t ; \tau, \bar{\beta})) \cdot \frac{\partial}{\partial \bar{\beta}} \beta(t ; \tau, \bar{\beta})=x_{\beta}(\tau, \bar{\beta})+\int_{\tau}^{t} u_{\beta}(s, \beta(s ; \tau, \bar{\beta})) \cdot \frac{\partial}{\partial \bar{\beta}} \beta(s ; \tau, \bar{\beta}) d s .
$$

Finally, differentiating w.r.t. $\bar{\beta}$ the identity (3.9), we obtain

$$
u_{\beta}(t, \beta(t ; \tau, \bar{\beta})) \cdot \frac{\partial}{\partial \bar{\beta}} \beta(t ; \tau, \bar{\beta})=u_{\beta}(\tau, \bar{\beta})+\int_{\tau}^{t} P_{x, \beta}(s, \beta(s ; \tau, \bar{\beta})) \cdot \frac{\partial}{\partial \bar{\beta}} \beta(s ; \tau, \bar{\beta}) d s .
$$

Combining (4.1)-(4.3), we thus obtain the system of ODEs

$$
\left\{\begin{aligned}
\frac{d}{d t}\left[\frac{\partial}{\partial \bar{\beta}} \beta(t ; \tau, \bar{\beta})\right] & =G_{\beta}(t, \beta(t ; \tau, \bar{\beta})) \cdot \frac{\partial}{\partial \bar{\beta}} \beta(t ; \tau, \bar{\beta}), \\
\frac{d}{d t}\left[x_{\beta}(t, \beta(t ; \tau, \bar{\beta})) \cdot \frac{\partial}{\partial \bar{\beta}} \beta(t ; \tau, \bar{\beta})\right] & =u_{\beta}(t, \beta(t ; \tau, \bar{\beta})) \cdot \frac{\partial}{\partial \bar{\beta}} \beta(t ; \tau, \bar{\beta}), \\
\frac{d}{d t}\left[u_{\beta}(t, \beta(t ; \tau, \bar{\beta})) \cdot \frac{\partial}{\partial \bar{\beta}} \beta(t ; \tau, \bar{\beta})\right] & =P_{x, \beta}(t, \beta(t ; \tau, \bar{\beta})) \cdot \frac{\partial}{\partial \bar{\beta}} \beta(t ; \tau, \bar{\beta}) .
\end{aligned}\right.
$$

In particular, the quantities within square brackets on the left hand sides of (4.4) are absolutely continuous. From (4.4), using Lemma 5 along a good characteristic we obtain

$$
\left\{\begin{aligned}
\frac{d}{d t} x_{\beta}+G_{\beta} x_{\beta} & =u_{\beta} \\
\frac{d}{d t} u_{\beta}+G_{\beta} u_{\beta} & =\left[u^{2}+\frac{1}{2}\left(u_{x}^{2}+\rho^{2}\right)-P\right] x_{\beta}=\left[u^{2}+\frac{1}{2}\left(\frac{1}{x_{\beta}}-1\right)-P\right] x_{\beta} \\
& =\left[u^{2}-P-\frac{1}{2}\right] x_{\beta}+\frac{1}{2}
\end{aligned}\right.
$$

3. We now go back to the original $(t, x)$ coordinates and derive an evolution equation for the partial derivative $u_{x}$ along a "good" characteristic curve. 
Fix a point $(\tau, \bar{x})$ with $\tau \notin \mathcal{N}$. Assume that $\bar{x}$ is a Lebesgue point for the map $x \mapsto u_{x}(\tau, x)$. Let $\bar{\beta}$ be such that $\bar{x}=x(\tau, \bar{\beta})$ and assume that $t \mapsto \beta(t ; \tau, \bar{\beta})$ is a good characteristic, so that (GC) holds. We observe that

$$
\left(u_{x}^{2}+\rho^{2}\right)(\tau, x)=\frac{1}{x_{\beta}(\tau, \bar{\beta})}-1 \geq 0 \quad x_{\beta}(\tau, \bar{\beta})>0 .
$$

4. (Equation of $\rho x_{\beta}$.) By equation of $\rho$, we have the weak formulation as

$$
\int_{x_{1}(t)}^{x_{2}(t)} \rho(t, x) d x-\int_{x_{1}(\tau)}^{x_{2}(\tau)} \rho(\tau, x) d x=0,
$$

where $x_{i}(i=1,2)$ are two characteristics, and denote $\beta_{i}$ as $x\left(t, \beta_{i}(t)\right)=x_{i}(t)(i=1,2)$. By changing of variable,

$$
\int_{\beta_{1}(t)}^{\beta_{2}(t)} \rho\left(t, x\left(t, \beta_{t}^{\prime}\right)\right) x_{\beta}\left(t, \beta_{t}^{\prime}\right) d \beta_{t}^{\prime}-\int_{\beta_{1}(\tau)}^{\beta_{2}(\tau)} \rho\left(\tau, x\left(\tau, \beta_{\tau}^{\prime}\right)\right) x_{\beta}\left(\tau, \beta_{\tau}^{\prime}\right) d \beta_{\tau}^{\prime}=0,
$$

Rewrite it as

$$
\int_{\beta_{1}(\tau)}^{\beta_{2}(\tau)} \rho\left(t, x\left(t, \beta_{t}^{\prime}\left(\beta_{\tau}^{\prime}\right)\right)\right) x_{\beta}\left(t, \beta_{t}^{\prime}\left(\beta_{\tau}^{\prime}\right)\right) \frac{d \beta_{t}}{d \beta_{\tau}} d \beta_{\tau}^{\prime}-\int_{\beta_{1}(\tau)}^{\beta_{2}(\tau)} \rho\left(\tau, x\left(\tau, \beta_{\tau}^{\prime}\right)\right) x_{\beta}\left(\tau, \beta_{\tau}^{\prime}\right) d \beta_{\tau}^{\prime}=0
$$

When $\left|\beta_{1}(\tau)-\beta_{2}(\tau)\right| \rightarrow 0, t-\tau \rightarrow 0$ we have

$$
\frac{d}{d t}\left[\rho\left(t, x\left(t, \beta_{t}^{\prime}\left(\beta_{\tau}^{\prime}\right)\right)\right) x_{\beta}\left(t, \beta_{t}^{\prime}\left(\beta_{\tau}^{\prime}\right)\right) \frac{d \beta_{t}\left(t, \beta_{\tau}\right)}{d \beta_{\tau}}\right]=0 .
$$

This implies

$$
\frac{d}{d t}\left(\rho x_{\beta}\right)\left(t, \beta\left(t, \beta_{\tau}\right)\right)=-\frac{d \beta}{d t}\left(\rho x_{\beta}\right)\left(t, \beta\left(t, \beta_{\tau}\right)\right)=-G_{\beta}\left(t, \beta\left(t, \beta_{\tau}\right)\right)\left(\rho x_{\beta}\right)\left(t, \beta\left(t, \beta_{\tau}\right)\right) .
$$

5. Let now $u=u(t, x)$ be a conservative solution. As shown by the previous analysis, in terms of the variables $t, \beta$ the quantities $x, u$ satisfy the semilinear system

$$
\left\{\begin{aligned}
\frac{d}{d t} \beta(t, \bar{\beta}) & =G(t, \beta(t, \bar{\beta})) \\
\frac{d}{d t} x(t, \beta(t, \bar{\beta})) & =u(t, \beta(t, \bar{\beta})) \\
\frac{d}{d t} x_{\beta}(t, \beta(t, \bar{\beta})) & =u_{\beta}(t, \beta(t, \bar{\beta}))-G_{\beta}(t, \beta(t, \bar{\beta})) x_{\beta}(t, \beta(t, \bar{\beta})) \\
\frac{d}{d t} u(t, \beta(t, \bar{\beta})) & =-P_{x}(t, \beta(t, \bar{\beta})) \\
\frac{d}{d t}\left(\rho x_{\beta}\right)(t, \beta(t, \bar{\beta})) & =-G_{\beta}(t, \beta(t, \bar{\beta}))\left(\rho x_{\beta}\right)(t, \beta(t, \bar{\beta})) \\
\frac{d}{d t} u_{\beta}(t, \beta(t, \bar{\beta})) & =\left(u^{2}(t, \beta(t, \bar{\beta}))-P(t, \beta(t, \bar{\beta}))\right) x_{\beta}(t, \beta(t, \bar{\beta})) \\
& +\frac{1}{2}\left(1-x_{\beta}(t, \beta(t, \bar{\beta}))\right)-G_{\beta}(t, \beta(t, \bar{\beta})) u_{\beta}(t, \beta(t, \bar{\beta})) .
\end{aligned}\right.
$$


We recall that $P$ and $G$ were defined at (1.2) and (3.11), respectively. The function $P$ admits a representation in terms of the variable $\beta$, namely

$$
\begin{gathered}
P(t, \beta)=\frac{1}{2} \int_{-\infty}^{\infty} \exp \left\{-\left|\int_{\beta}^{\beta^{\prime}} x_{\beta}(s) d s\right|\right\} \cdot\left[u^{2}\left(\beta^{\prime}\right) x_{\beta}\left(\beta^{\prime}\right)+\frac{1}{2}\left(1-x_{\beta}\left(\beta^{\prime}\right)\right)\right] d \beta^{\prime}, \\
P_{x}(t, \beta)=\frac{1}{2}\left(\int_{\xi}^{\infty}-\int_{-\infty}^{\xi}\right) \exp \left\{-\left|\int_{\xi}^{\beta^{\prime}} x_{\beta}(s) d s\right|\right\}\left[u^{2}\left(\beta^{\prime}\right) x_{\beta}\left(\beta^{\prime}\right)+\frac{1}{2}\left(1-x_{\beta}\left(\beta^{\prime}\right)\right)\right] d \beta^{\prime}, \\
G(t, \beta) \doteq \int_{-\infty}^{\beta}\left[\frac{u_{\beta}\left(\beta^{\prime}\right)}{x_{\beta}\left(\beta^{\prime}\right)}+\left(2 u^{2}\left(\beta^{\prime}\right)-2 P\left(\beta^{\prime}\right)\right) \frac{u_{\beta}\left(\beta^{\prime}\right)}{x_{\beta}\left(\beta^{\prime}\right)}\right] x_{\beta}\left(\beta^{\prime}\right) d \beta^{\prime} \\
G_{\beta}(t, \beta)=u_{\beta}\left(1+2 u^{2}-2 P\right) .
\end{gathered}
$$

For every $\bar{\beta} \in \mathbb{R}$ we have the initial condition

$$
\left\{\begin{aligned}
\beta(0, \bar{\beta}) & =\bar{\beta}, \\
x(0, \bar{\beta}) & =x(0, \bar{\beta}), \\
x_{\beta}(0, \bar{\beta}) & =x_{\beta}(0, \bar{\beta}), \\
\left(\rho x_{\beta}\right)(0, \bar{\beta}) & =\rho_{0}(x(\bar{\beta})) x_{\beta}(0, \bar{\beta}), \\
u(0, \bar{\beta}) & =u_{0}(x(0, \bar{\beta})), \\
u_{\beta}(0, \bar{\beta}) & =u_{0, \beta}(x(0, \bar{\beta})) .
\end{aligned}\right.
$$

Note that $\rho_{0}$ is in $\left(\rho x_{\beta}\right)(0, \bar{\beta})$. By the Lipschitz continuity of all coefficients, the Cauchy problem (4.11), (4.16) has a unique solution, globally defined for all $t \geq 0, x \in \mathbb{R}$.

6. To complete the proof of uniqueness, consider two conservative solutions $u, \tilde{u}$ of the twocomponent Camassa-Holm equation (1.1) with the same initial data $u_{0} \in H^{1}(\mathbb{R}), \rho_{0} \in L^{2}(\mathbb{R})$. For a.e. $t \geq 0$ the corresponding Lipschitz continuous maps $\beta \mapsto x(t, \beta), \beta \mapsto \tilde{x}(t, \beta)$ are strictly increasing. Hence they have continuous inverses, say $x \mapsto \beta^{*}(t, x), x \mapsto \tilde{\beta}^{*}(t, x)$.

By the previous analysis, the map $(t, \beta) \mapsto\left(x, u, x_{\beta},\left(\rho x_{\beta}\right), u_{\beta}\right)(t, \beta)$ is uniquely determined by the initial data $\rho_{0}, u_{0}$. Therefore

$$
x(t, \beta)=\tilde{x}(t, \beta), \quad u(t, \beta)=\tilde{u}(t, \beta) .
$$

In turn, for a.e. $t \geq 0$ this implies

$$
u(t, x)=u\left(t, \beta^{*}(t, x)\right)=\tilde{u}\left(t, \tilde{\beta}^{*}(t, x)\right)=\tilde{u}(t, x) .
$$

Then we can get the uniqueness of $\rho$ by

$$
\rho(t, x)=\rho\left(t, \beta^{*}(t, x)\right)=\frac{\left(\rho x_{\beta}\right)\left(t, \beta^{*}(t, x)\right)}{x_{\beta}\left(t, \beta^{*}(t, x)\right)}=\frac{\left(\rho x_{\beta}\right)\left(t, \beta^{*}(t, x)\right)}{x_{\beta}\left(t, \beta^{*}(t, x)\right)}=\tilde{\rho}\left(t, \beta^{*}(t, x)\right)=\tilde{\rho}(t, x),
$$

whenever $x_{\beta} \neq 0$. And considering that the set $\left\{(t, x): x_{\beta}(t, \beta)=0\right\}$ is a measure zero set, so $\rho$ is uniquely defined almost everywhere.

Acknowledgement. This work was done when M. J. Li was visiting the Mathematics Department of The Pennsylvania State University, whose hospitality is gratefully acknowledged. The authors would thank R. M. Chen for helpful discussion. M. J. Li is supported by the NSFC Grant No. 11201503, the Fundamental Research Funds for the Central Universities No. 2014MDLXYXJ02 and the State Scholarship Fund, Scholarship Council of China. Q. Zhang is partially supported by NSF grant DMS-1411786. 


\section{References}

[1] A. Bressan, G. Chen and Q. Zhang, Uniqueness of conservative solutions to the CamassaHolm equation via characteristics. Discr. Cont. Dyn. Syst. 35 (2015), 25-42.

[2] A. Bressan, G.Chen and Q.Zhang, Unique solutions to a variational wave equation Arch. Rational Mech. Anal., to appear.

[3] A. Bressan and A. Constantin, Global conservative solutions to the Camassa-Holm equation, Arch. Rat. Mech. Anal. 183 (2007), 215-239.

[4] A. Bressan and A. Constantin, Global dissipative solutions to the Camassa-Holm equation, Analysis and Applications 5 (2007), 1-27.

[5] A. Bressan and M. Fonte, An optimal transportation metric for solutions of the CamassaHolm equation, Methods and Applications of Analysis, 12 (2005), 191-220.

[6] R. Camassa and D. D. Holm, An integrable shallow water equation with peaked solitons, Phys. Rev. Lett. 71 (1993), 1661-1664.

[7] R.M. Chen, Y. Liu, Wave breaking and global existence for a generalized two-component Camassa-Holm system. Int. Math. Res. Not. 6 (2011) 1381-1416.

[8] A. Constantin, R.I. Ivanov, On an integrable two-component Camassa-Holm shallow water system. Phys. Lett. A 372(2008),7129-7132.

[9] C. Dafermos, Generalized characteristics and the Hunter-Saxton equation. J. Hyperbolic Diff. Equat. 8, (2011), 159-168.

[10] J. Escher, O. Lechtenfeld, Z. Yin, Well-posedness and blow-up phenomena for the 2component Camassa-Holm equation. Discrete Contin. Dyn. Syst. 19 (2007),493-513.

[11] Y. Fu, Y. Liu, C. Qu, Well-posedness and blow-up solution for a modified two-component periodic Camassa-Holm system with peakons.Math. Ann. 348 (2010), no. 2, 415-448.

[12] L. C. Evans, Partial Differential Equations. Second edition. American Mathematical Society, Providence, RI, 2010.

[13] K. Grunert, Blow-up for the two-component Camassa-Holm system.Discrete Contin. Dyn. Syst. 35 (2015) 2041-2051.

[14] K. Grunert, H. Holden and X. Raynaud, Global solutions for the two-component Camassa-Holm system, Comm. Partial Differential Equations 37 (2012), 2245-2271.

[15] K. Grunert, H. Holden, and X. Raynaud, A continuous interpolation between conservative and dissipative solutions for the two-component Camassa-Holm system. Forum Math. 3 (2015) 73 pages.

[16] C. Guan, Z. Yin, Global existence and blow-up phenomena for an integrable twocomponent Camassa-Holm shallow water system.J. Diff. Eqs. 248 (2010), 2003-2014.

[17] C. Guan, Z. Yin, Global weak solutions for a two-component Camassa-Holm shallow water system. J. Funct. Anal. 260 (2011) 1132-1154. 
[18] G. Gui, Y. Liu, On the global existence and wave breaking criteria for the two-component Camassa-Holm system. J. Func. Anal. 258 (2010) 4251-4278.

[19] G. Gui, Y. Liu, On the Cauchy problem for the two-component Camassa-Holm system. Math. Z. 268 (2011) 45-66.

[20] H. Holden and X. Raynaud, Global conservative solutions of the Camassa-Holm equation: a Lagrangian point of view. Comm. Partial Diff. Equat. 32 (2007), 1511-1549.

[21] Z. Xin and P. Zhang, On the weak solutions to a shallow water equation, Comm. Pure Appl. Math. 53 (2000), 1411-1433.

[22] Z. Xin and P. Zhang, On the uniqueness and large time behavior of the weak solutions to a shallow water equation, Comm. Partial Diff. Equat. 27 (2002), 1815-1844. 